died later, and no fewer than 20 died within three months of diagnosis. The commonest cause of death was haemorrhage in the skin, usually from mucous membranes; sometimes it was cerebral as well. They treated their patients with blood transfusion and prednisolone. "The key to effective management," they said, " is to give repeated blood transfusions to keep the haemoglobin level, if possible, between $60-70 \%$," and they described how this could be done by organizing out-patient sessions for blood transfusion. Prednisolone was given since it is known to reduce fragility of the capillaries and so help to reduce haemorrhage, and fluoxymesterone was used to control menorrhagia, almost always a serious complication in the women patients.

- At page 1027 of this issue of the B.M.F. Dr. S. M. Lewis describes experience at the Postgraduate Medical School, London, with 60 patients seen over the last 12 years. Twentyeight had a history of recent exposure to a possibly toxic drug, chloramphenicol and phenylbutazone being again the most frequent. The diagnosis was confirmed in all but two cases by aspiration of bone-marrow, but he points out that more than one aspiration may be needed to give a true picture. Within 15 months half the patients were dead and there was little difference in survival between patients in the toxic and idiopathic groups. In Dr. Lewis's patients, as in other groups, haemorrhage has been the main danger. $\mathrm{He}$ also has some patients who have survived for long periods and apparently recovered. Like other workers, Dr. Lewis relies on repeated transfusion for treatment and considers that transfusion of platelets may be helpful. In addition to steroids he has given androgens, as recommended by $N$. T. Shahidi and L. K. Diamond. ${ }^{6}$ They seemed to be beneficial in childhood near puberty but less so to adult patients. The benefit they gave seemed to last only a short time and neither they nor steroids had much influence on the prognosis. In a disease like aplastic anaemia which has a natural course punctuated by spontaneous remissions and unheralded relapses prognosis is indeed difficult to assess. Dr. Lewis considers that the more severe the pancytopenia the graver the risk of early death, and the longer the patient survives the better the chance of ultimate remission and perhaps cure. Like his predecessors he regards persistent and severe thrombocytopenia as the gravest risk, more serious than anaemia or neutropenia.

From these reports it is clear that aplastic anaemia must still be regarded as a grave condition, though there is perhaps a $25 \%$ chance of ultimate complete recovery. Repeated blood transfusion remains the most important therapeutic measure. Most physicians also give steroids, usually in the form of prednisolone in moderately large doses (50 to 75 mg. daily or the one-tenth equivalent as betamethasone). The value of androgens to stimulate erythropoiesis remains doubtful ; successful results have nearly all been recorded in children. Some benefit has been reported with oxymethalone. ${ }^{7}$ J. G. Humble ${ }^{8}$ has suggested the use of phytohaemagglutinin. This is an extract from the seeds of the bean Phaseolus vulgaris, which contains mucoproteins and is used in tissue culture for its property of causing human lymphocytes to enlarge and undergo active mitotic division. Six patients were treated by intravenous administration, and some evidence of stimulation of marrow was seen in all of them; in two the peripheral blood picture returned to normal. This form of treatment is still under trial, and more recent reports ${ }^{3}$ have been less enthusiastic.

Once aplastic anaemia is diagnosed, treatment should be given as soon as possible. Though the prognosis is grave, the disease is not necessarily fatal, and the longer the patient survives the better his or her chances of eventual recovery. The physician can help to prevent the disease by taking special care over prescribing drugs apt to cause it, and in particular ensuring that when they are indicated they are given in short courses for specific purposes.

\section{Electrical Stimulation of the Bladder}

Electrical excitability of the bladder was described by C. C. Stewart as early as $1900 .^{1} \quad$ The recent success of long-term cardiac pacemaking has revived interest in the feasibility of similar stimulation of the bladder in paraplegic patients to empty it efficiently. In animals the bladder has been stimulated either directly with percutaneous wires, ${ }^{23}$ or indirectly with induction coils ${ }^{34}$ or with implanted radiofrequency receivers. ${ }^{2-7}$ W. W. L. Glenn and his co-workers, using paraplegic dogs, stimulated the sacral nerve-roots bilaterally, the fundal branches of the vesical nerves bilaterally, and the bladder wall itself. They found that, though a smaller voltage was needed to empty the bladder by stimulation of the sacral or vesical nerves than by direct excitation of the bladder, fewer impulses spread to adjacent structures from direct stimulation than from nerve stimulation. A. Kantrowitz and $M$. Schamaun ${ }^{2}$ showed that in paraplegic dogs the bladder could be emptied completely by direct stimulation for two or three weeks after cord transection, but that later there was an increasingly high volume of residual urine in spite of a satisfactory rise in bladder pressure. After bilateral pudendal neurectomy the bladder once again completely emptied by electrical stimulation.

Implantation of radiofrequency receivers into patients with paraplegia has already been attempted, the largest published series being that of W. E. Bradley and his colleagues. ${ }^{6}$ Electrodes were implanted into the bladder wall of five paraplegic or quadriplegic patients. The electrodes were connected to receivers implanted under the fascia of the abdominal muscles, and the antenna of the transmitter was placed near the skin directly over the receiver. Contraction of the bladder was achieved in all five patients, but in only two cases was this accompanied by voiding of urine. In one of these two cases the unit failed later and in the other it had to be removed after two weeks because of infection.

There are still many problems to be overcome before electrical control of micturition can be used in the treatment of dysfunction of the bladder. The main ones are pain produced by stimulation, and the spread of nervous impulses to adjacent structures-especially the pelvic floor and the rectum. Early failure of the apparatus or of the electrodes is still common, and infection and rejection of the apparatus

\footnotetext{
${ }^{1}$ Stewart, C. C., Amer. F. Physiol., 1900, 4, 185.

Kantrowitz, A., and Schamaun, M., F. Amer. med. Ass., 1964, 187, 595

Boyce, W. H., Lathem, J. E., and Hunt, L. D., F. Urol. (Baltimore), 1964, 91, 41 .

+ Burgheie, T., Ichim, V., and Demetresco, M., F. Urol. míd. chir., 1958, 64, 317 .

${ }^{5}$ Bradley, W. E., Wittmers, L. E., Chou, S. N., and French, L. A., f. Neurosurg., 1962, 19, 782.

6 Chou, S. N., and French, L. A., ibid., 1963, 20, 953.

Glenn, W. W. L., Hageman, J. H., Mauro, A., Eisenberg, L., Flanigan, S., and Harvard, M., Ann. Surg., 1964, 160, 338.

${ }^{8}$ Guttman, L., and Whitteridge, D., Brain, 1947, 70, 361.

${ }^{9}$ Caldwell, K. P. S., Lancet, 1963, 2, 174.
} 
must be expected in many cases. There is a possibility of serious cardiovascular reactions in patients with spinal-cord lesions at and above D5 segment, especially in cervical lesions. ${ }^{\circledR}$ Moreover, a neurogenic bladder, whether automatic or autonomous, represents a multitude of complex functional problems, and electrical control of part of the intermittent function of the bladder is unlikely to give both efficient emptying and continence. This also applies to the method suggested by K. P. S. Caldwell ${ }^{9}$ of implanting radio-controlled electrodes into the external urethral sphincter. Possibly this method may give a degree of continence to patients suffering from incontinence-especially female patients, for whom there is still no effective urinal available-but at present it remains a research technique.

\section{Fits in Childhood}

Convulsive attacks in childhood are surprisingly common. Dr. J. E. Cooper in the B.M.F. this week (p. 1020) reports that of the 5,000 children who form the National Survey of Health and Development just over $2 \%$ had had a fit or fits during the first two years of life. By the age of 5 years, according to F. J. W. Miller ${ }^{1}$ and his colleagues in Newcastle upon Tyne, $7.2 \%$ of the children in the "Thousand Family Survey" had had one convulsion or more, and $20 \%$ of these children had died. All the deaths except one occurred under the age of 1 year. Three deaths were attributed to the effects of birth trauma, three infants died in the first three months of life from unknown causes, and one child with recurrent unexplained fits died at the age of 7 months. The other five deaths were associated with infections, two with meningitis, two with gastro-enteritis, and one with a probable encephalitis. Complacency about the management of children with fits is not justified.

The main difficulties arise when dealing with a first fit, though the treatment during the fit itself will be the same whether it is one of a series or a totally unexpected event. Certain principles need emphasizing. If the child is seen while still in a convulsion the immediate consideration is to prevent damage to the brain from the hypoxia likely to occur in a prolonged fit. Provision of an adequate airway is more important than the traditional concern about biting the tongue. The child should be placed on his side with the head lowered rather than raised; secretions should be sucked out of the pharynx, and a pharyngeal airway should be inserted (pharyngeal airways of various sizes should be in any emergency bag). The next thing is to attempt to stop the convulsion by the injection of a sedative: soluble sodium phenobarbitone is probably the most useful and convenient drug and should be given intramuscularly in a dose of 30 mg. ( $\frac{1}{2}$ gr.) for a child under 1 month, $60 \mathrm{mg}$. ( $1 \mathrm{gr}$.) between 1 month and under 1 year, $120 \mathrm{mg}$. ( $2 \mathrm{gr}$.) between 1 and 3 years, and $120-180 \mathrm{mg}$. (2-3 gr.) over 3 years of age. A rapid clinical examination should be made to determine the cause of the convulsion. If the child has not had a previous convulsion he should be transferred to hospital as soon as he is fit to travel, accompanied by a note giving the time of injection of the sedative and the dose given.

More commonly the child is seen after the fit. If it is a first fit admission to hospital should be arranged, and for infants an injection of soluble phenobarbitone should be given in half the dose suggested above; for older children immediate sedation is usually unnecessary just after the seizure.

The common causes of convulsions are different at different age groups. In the first few weeks of life fits are usually due to one of three causes-cerebral damage related to birth (hypoxia or intracranial haemorrhage), infection (meningitis or septicaemia), or developmental abnormalities of the brain. Febrile convulsions are extremely rare in the newborn. A precise diagnosis based on clinical examination alone may be difficult or impossible without a lumbar puncture. The infant with cerebral damage or meningitis is usually irritable and may have a bulging fontanelle. When a cerebral malformation is present the child may be apathetic and inert, and there may be an obvious abnormality such as microcephaly or a facial port-wine naevus (Sturge-Weber syndrome). In all three categories urgent admission to hospital is required. A small group of cases remains in which the fit is brief and the child appears normal between the seizures: in these circumstances the advice of a paediatrician should be obtained as soon as possible. In some of these infants hypocalcaemic tetany is the cause of the convulsions. It affects almost exclusively infants fed on cow's milk and is commonest in the first week of life. Finally, there will be a small proportion of babies in whom the convulsion is simply the first of a long series of epileptic attacks. Idiopathic epilepsy should be diagnosed only after rigorous exclusion of other possible causes.

After the neonatal period the first fit usually occurs in a previously normal child. The majority of these children have an infection. A clear distinction must be made between the following: a fit due to disease of the meninges or brain, as in meningitis or encephalitis; a fit which is related to a rapid rise in temperature due to an infection not involving the brain ; and a fit due to sagittal-sinus thrombosis or severe electrolyte disturbance, both of which may occur in gastroenteritis. It is frequently impossible without observation over the course of a number of attacks and by using electroencephalography to distinguish between genuine "febrile convulsions" and recurrent epileptic attacks triggered off by a high temperature. Certain factors are helpful in making the distinction-and it is one that should be made. In many cases of "febrile convulsions" there is a family history of similar attacks ceasing at the age of 6 or 7 years, and the electroencephalogram is usually normal. In making a diagnosis $\mathrm{S}$. Livingstone ${ }^{2}$ considers that the following points favour epilepsy: a generalized or focal fit lasting longer than one hour, a focal convulsion of any duration, a history of proved epilepsy in the immediate family, and an abnormal electroencephalogram.

The recognition of convulsive seizures in childhood is not always simple. In the neonatal period a tonic fit may be mistaken for an apnoeic or cyanotic attack, but careful observation will show that in a fit some jerking and twitching occurs and that the cyanosis is due to interference with respiration; in an apnoeic attack the anoxia may cause a few convulsive movements at the end of the attack. At the toddler age breath-holding attacks are often mistaken for fits. A detailed history will usually indicate the circumstances of rage or frustration in which they occur. Masturbation is

\footnotetext{
1 Miller, F. J. W., Court, S. D. M., Walton, W. S., and Knox, E. G. Growing up in Newcastle upon Tyne, 1960, pp. 164-173. Published for the Nuffield Foundation by the Oxford University Press, London.

2 Livingstone, S., The Diagnosis and Treatment of Convulsive Disorders in Children, 1954, p. 80. Thomas, Springfield, Illinois.
} 